\title{
Risk Factors and Response to Treatment of Chronic Migraine Patient
}

\author{
(D) Barış Kıran¹, (D) Onur Akan², (1D Serap Üçler² \\ ${ }^{1}$ Çan State Hospital, Clinic of Neurology, Çanakkale, Turkey \\ 2 University of Health Sciences Turkey, Prof. Dr. Cemil Taşçığlu City Hospital, Clinic of Neurology, Istanbul, Turkey
}

\section{Abstract}

Objective: Migraine is a common cause of medical consultation in neurology policlinics. Chronic migraine usually develops as a complication of episodic migraine. It causes significant distress with substantial impact on the quality of life of an individual and huge economic cost to the society through occupational disability and healthcare consultations. Chronic migraine and medication-overuse headache often coexist. The aim of this study was to determine the risk factors for the transformation to chronic migraine and treatment for chronic migraine.

Methods: One hundred thirty-nine patients diagnosed with chronic migraine between January 2014-January 2015 were included in the study. These patients were observed for risk factors and prophylactic treatment.

Results: $92.8 \%$ of the patients in the study were female and were between the ages of $18-63.85 \%$ of patients had headache for more than five years. Risk factors for the transformation from episodic to chronic migraine were determined as low-level of education, low-middle social economic status, emotional stress, obesity and sleep disorders. All patients were treated with prophylactic treatment. 19 patients were not evaluated because they failed to follow the protocol of treatment. $72 \%$ of patients responded to treatment and $27.5 \%$ of patients failed treatment. It was determined that only the accompanying depression had a negative effect on treatment response.

Conclusion: In our study, female gender, low educational level, low-middle socioeconomic level, obesity, preobesity, comorbid psychiatric disease and analgesic overuse have been shown as risk factors for the transformation from episodic to chronic migraine. Prophylactic drugs used in the treatment of episodic migraine had similar rates of response to treatment when used in chronic migraine prophylaxis. Treatment with botulinum toxin $A$ and dry needle therapy used in the treatment-resistant patient has been shown to significantly increase treatment compliance and response. The presence of depression has been shown to negatively affect treatment.

Keywords: Chronic migraine, risk factors, treatment

\section{INTRODUCTION}

Migraine is a multifactorial neurovascular syndrome characterized by episodes of headache that occur with various triggering factors in people with genetic sensitivity. For three months and more, headache for 15 days a month, at least 8 of which are of migraine character, is chronic migraine (1). The prevalence of migraine is about $15-20 \%(2,3)$. Migraine is chronic at a rate of $4 \%$ per year (4). The prevalence of chronic migraine in society is about $2 \%$ (5). Chronic migraine causes serious deterioration in the quality of life and economic loss of patients $(6,7)$. Patients develop a high rate of relapse after treatment, which is a problem in treatment and follow-up (8). Various risk factors facilitate the transformation of episodic migraine (9-13). In this study, we aimed to determine the risk factors that facilitate chronic pain in patients diagnosed with chronic migraine and to analyze treatment responses.

\section{METHODS}

In January 2014-January 2015, patients aged 18-65 who were diagnosed with chronic migraine were included in this study 
retrospectively in the neurology clinic headache outpatient clinic of the Ministry of Health Okmeydanı Training and Research Hospital. Our study was approved by the Ethics Committee of Saglık Bilimleri University Okmeydanı Training and Research Hospital dated 14.04.2015 and numbered 303. Demographic findings, causes that may be risk factors for chronic pain, accompanying comorbidities, causes that predispose pain, Hamilton depression scales were examined.

Migraine treatments applied to patients were recorded from patient files. The risk factors that may lead to chronic headache were examined. Their responses to different treatments were analyzed and the effect of risk factors on treatment response was evaluated.

\section{Statistical Analysis}

Descriptive statistics are presented as mean, standard deviation and percentage. The mean of the quantitative data was compared with the t-test, while the broad properties of the ordinal and variance were compared with the Mann-Whitney $\mathrm{U}$ test. The categorical data was evaluated with chi-square or Fisher tests and the significance level of $p<0.05$ was accepted in all tests.

\section{RESULTS}

One hundred thirty-nine patients were enrolled in the study. Of the patients, 129 (92.8\%) were female and 10 (7.2\%) were male. The mean age of the patients was 41.5 \pm 10.8 . Demographic findings on age, duration of headache, duration of migraine diagnosis, duration of chronic headache and number of days of headache per month are summarized in Table 1.

$77.7 \%(n=108)$ of the patients were married and $28.7 \%(n=40)$ were working in a regular job. $13.7 \%$ of patients $(n=19)$ stated that their income was poor, while $77 \%(n=107)$ stated that they were normal (based on the minimum wage for 2015). In $88.5 \%$ of patients ( $n=123$ ) headache onset age was under 40 years of age, total headache duration ranged between 8-480 months, mean 169.2 months, migraine diagnosis period ranged between 0-480 months, mean 80.8 months and average number of head pain days per month was $23.9 \pm 5.7$ days/month. The visual analog scale score of $83.4 \%(n=116)$ of the patients was 8 and above. $61.9 \%(n=86)$ of the patients had primary school and below education level. $41.7 \%(n=58)$ were preobes [body mass index (BMI): $24-30 \mathrm{~kg} / \mathrm{m}^{2}$ ], $28.8 \%(\mathrm{n}=40)$ were obese (BMI: $30-40 \mathrm{~kg} /$ $\left.\mathrm{m}^{2}\right)$ and $2.9 \%(\mathrm{n}=4)$ were morbidly obese. $31.7 \%$ of the patients were over the obesity limit and $73.4 \%$ were overweight and above. The average number of applications to the emergency department in the last year was 7.07 days/year. $81.3 \%$ of patients had been admitted to multiple centres for headaches. While 43.9\% ( $n=61)$ had no comorbidity, 20.1\% ( $n=28)$ had more than one comorbidity. The accompanying comorbidities are indicated in Table 2.

$71.2 \%$ of patients ( $n=99$ ) had overuse of analgesics. Overused analgesics were $41.7 \%(n=58)$ non-steroidal anti-inflammatory (NSAIDs), 10.8\% $(n=15)$ ergotamine, $1.4 \%(n=2)$ triptan and $17.3 \%$ different combinations of these drugs according to their frequency. Prophylactic treatment was initiated for all patients enrolled in the study. Because 19 patients did not continue follow-up, the treatment response could not be assessed. 50\% and above decrease in pain frequency after taking prophylactic treatment at appropriate dose for at least 3 months was evaluated as a positive response to the treatment. $72.5 \%(n=88)$ of the follow-up patients received response to the treatment. The responses of patients to different treatment protocols are summarized in Table 3.

The effects of demographic findings, risk factors, accompanying comorbid diseases, pain predisposing factors and clinical findings

\begin{tabular}{|l|l|l|l|}
\hline \multicolumn{3}{|l|}{ Table 1. Demographic findings of patients (n=139) } \\
\hline Parameters & Minimum & Maximum & Mean \\
\hline Age & 18 & 64 & $41.5 \pm 10.8$ \\
\hline $\begin{array}{l}\text { Headache duration } \\
\text { (months) }\end{array}$ & 8 & 480 & $169.2 \pm 114.6$ \\
\hline $\begin{array}{l}\text { Diagnosis of migraine } \\
\text { (month) }\end{array}$ & 0 & 480 & $80.8 \pm 92.4$ \\
\hline $\begin{array}{l}\text { Chronic headache duration } \\
\text { (months) }\end{array}$ & 2 & 420 & $53.8 \pm 66.7$ \\
\hline $\begin{array}{l}\text { Number of head pain days } \\
\text { per month }\end{array}$ & 15 & 30 & $23.9 \pm 5.7$ \\
\hline
\end{tabular}

\begin{tabular}{|l|l|l|l|}
\hline \multicolumn{4}{|l|}{ Table 2. Accompanying comorbidities } \\
\hline Disease & Frequency & Disease & Frequency \\
\hline $\begin{array}{l}\text { Gastrointestinal } \\
\text { disease }\end{array}$ & $\begin{array}{l}\% 13 \\
(\mathrm{n}=18)\end{array}$ & Liver disease & $\begin{array}{l}\% 3.6 \\
(\mathrm{n}=5)\end{array}$ \\
\hline Hypertension & $\begin{array}{l}\% 12.2 \\
(\mathrm{n}=17)\end{array}$ & Cardiac disease & $\begin{array}{l}\% 3.6 \\
(\mathrm{n}=5)\end{array}$ \\
\hline Thyroid disease & $\begin{array}{l}\% 12.2 \\
(\mathrm{n}=17)\end{array}$ & Nephrolithiasis & $\begin{array}{l}\% 2.9 \\
(\mathrm{n}=4)\end{array}$ \\
\hline Asthma & $\begin{array}{l}\% 9.4 \\
(\mathrm{n}=13)\end{array}$ & Allergy (other) & $\begin{array}{l}\% 2.2 \\
(\mathrm{n}=3)\end{array}$ \\
\hline Psychiatric illness & $\begin{array}{l}\% 8.6 \\
(\mathrm{n}=12)\end{array}$ & Epilepsy & $\begin{array}{l}\% 2.2 \\
(\mathrm{n}=3)\end{array}$ \\
\hline $\begin{array}{l}\text { Iron deficiency } \\
\text { anemia }\end{array}$ & $\begin{array}{l}\% 6.5 \\
(\mathrm{n}=9)\end{array}$ & $\begin{array}{l}\text { Vitamin B12 } \\
\text { deficiency } \\
\text { anemia }\end{array}$ & $\begin{array}{l}\% 1.4 \\
(\mathrm{n}=2)\end{array}$ \\
\hline Diabetes mellitus & $\begin{array}{l}\% 3.6 \\
(\mathrm{n}=5)\end{array}$ & & \\
\hline
\end{tabular}




\begin{tabular}{|l|l|l|l|}
\hline \multicolumn{4}{|l|}{ Table 3. Prophylactic treatment responses } \\
\hline Treatment & Response & No response & $\begin{array}{l}\text { Side effect } \\
\text { developed }\end{array}$ \\
\hline Propranolol & $\% 42.9(n=6)$ & $\% 57.1(n=8)$ & - \\
\hline Flunarizine & $\% 45.9(n=28)$ & $\% 34.4(n=21)$ & $\% 19.7(n=12)$ \\
\hline Topiramate & $\% 54(n=34)$ & $\% 33.3(n=21)$ & $\% 12.7(n=8)$ \\
\hline Sodium valproate & $\% 37.5(n=6)$ & $\% 50(n=8)$ & $\% 12.5(n=2)$ \\
\hline Venlafaxine & $\% 60(n=21)$ & $\% 31.4(n=11)$ & $\% 8.6(n=3)$ \\
\hline Amitriptyline & $\% 39.1(n=9)$ & $\% 39.1(n=9)$ & $\% 21.7(n=5)$ \\
\hline SSRI & $\% 23.1(n=6)$ & $\% 69.2(n=18)$ & $\% 7.7(n=2)$ \\
\hline Pregabalin & $\% 28.6(n=2)$ & $\% 28.6(n=2)$ & $\% 42.9(n=3)$ \\
\hline Dry needle & $\% 51.9(n=14)$ & $\% 48.1(n=13$ & - \\
\hline Botulinum toxin A & $\% 70.1(n=25)$ & $\% 21.9(n=7)$ & - \\
\hline Total & $\% 72.5(n=88)$ & $\% 27.5(n=32)$ & \\
\hline SSRI: Serotonin reuptake inhibitor & & \\
\hline
\end{tabular}

on treatment responses were examined. After this examination, it was observed that only the presence of psychiatric illness negatively affected the treatment response. In $21.9 \%(n=7)$ of the patients who did not receive treatment response, this rate was $5.7 \%(n=5)$ while there was a history of psychiatric illness. Statistically, patients with a history of psychiatric illness responded worse to treatment $(p=0.015)$.

\section{DISCUSSION}

According to the definition of the International Headache classification-3, chronic migraine is a headache of 15 days per month and above, with at least 8 of which are migratory in character (1) for more than 3 months. It occurs in about $2 \%$ of the world's population and more in women. The transformation of episodic migraine to chronic migraine is the transformation of migraine, which usually develops gradually. Episodic migraines develop into chronic migraines at a rate of about $4 \%$ per year. The transformation of migraine is not absolute irreversible, remission can be achieved by spontaneous and/or treatment. Chronic migraines lead to more serious losses in quality of life than episodic migraines, and consequent economic losses. Nonmodifiable risk factor for the transformation of episodic migraine and chronic migraine are gender, genetic background, history of head and neck injury, low education level, low socioeconomic status, a lot of stress, starting in a young age, initially high headache frequency, presence of concomitant psychiatric illness and allodynia. The modifiable risk factors for the transformation are snoring, excessive caffeine consumption, obesity, sleep disorders and overuse of analgesics (14). Determining the modifiable risk factors that play a role in the transformation of migraine is effective on treatment responses and the course of the disease.

Migraines and chronic migraines are more common in women. Female sex is thought to be a risk factor for migraine, but it is controversial to think of it as a risk factor in terms of chronic headache. Scher et al. (12) in a study comparing chronic daily headache with episodic headache, the proportion of women with chronic migraines was found to be higher than that of women with episodic migraines. In the Castillo study of 1883 chronic migraine patients, the ratio of male/female was 6.5/1 (15), in the Greater Taipei study of 3377 chronic migraine patients, the ratio was 4.5/1 (16), and in the Baltimore study of 13343 chronic migraine patients, the ratio was 2.5/1 (17). In our study, the female/male ratio of 12.9/1 indicated the predominance of female sex in chronic migraine in line with the literature.

Migraine is most commonly seen in the 30-40 age range and its incidence decreases as age progresses. In a study conducted by Scher et al. (12), it was determined that the frequency of chronic headaches decreased in later ages. According to the same study, $29 \%$ of patients with episodic head pain were transformed into chronic headache at the first 1 week after the onset of headache, $8 \%$ at 1 week 1 month, $35 \%$ at 1 month- 10 years, and $28 \%$ at more than 10 years. In our study, the mean age of the patients was $41.5 \pm 10.8$ and $88.5 \%$ had headache onset under the age of 40 . In $13 \%$ of patients, headaches were chronic in the first 1 month, in $50 \%$ in 1 month 10 years and in $37 \%$ in 10 years and above. This difference in chronic periods may be due to sociocultural and anthropological differences belonging to our society.

Although clinical studies show that migraine develops more often in people with high intellectual and educational levels, community-based studies do not confirm this. Some studies suggest that there may be an increase in the prevalence of headaches with low income status. In a Norwegian study, 22718 patients with no initial headache were followed for 11 years, and the prevalence of high frequency headache and chronic headache was higher in patients with low educational and low socioeconomic levels (18). In a study conducted by Scher et al. (12), those with lower level education than high school were found to be 3 times more risky in terms of chronic headache development than those with higher level education and university education. In our study, in line with the literature, $90.6 \%$ of the patients had low-to-medium income status and $61.9 \%$ had primary and lower education levels.

Although some studies have shown that chronic migraine is not associated with BMI, numerous epidemiological and clinical studies have shown that underweight, preobesity and obesity are 
risk factors for chronic migraine. According to the meta-analysis, preobesite and obesity, especially in female sex, were thought to be risk factors for chronic migraine $(19,20)$.

In the review of Bigal et al. (6) the risk of developing chronic daily headache was 5 times higher in patients with BMI over 30. In three broad epidemiological studies, the effect of BMI on migraine progression was evaluated; in the first study, high frequency migraines were developed in $4.4 \%$ of normal-weight patients, while in preobesis group, this ratio was found to be $5.8 \%$ and $13.6 \%$ in obese patients. This study showed that preobesity and obesity lead to increased risk in the transformation of episodic migraine to high-frequency migraine. In the second study, obesity led to a higher risk increase in chronic migraines than in chronic tension-type headaches. In another study, the prevalence of chronic migraine was found to be $0.9 \%$ in normal weight and 3.2\% in obese people (21). The results of these studies prove the effect of obesity on migraine progression. In a study by Tietjen et al. (22) on 721 migraine patients, headache frequency and headache related disability rates were found to be significantly higher in the group of patients with obesity and depression compared to the group without obesity and depression, and also in the accompanying anxiety disorder, similar results were obtained. According to the results of the depression scale, the presence of depression in all patients was $62.6 \%$, whereas in the group of patients above the obesity limit, this ratio was $72.7 \%$. These results support the tight relationship between depression and obesity.

Chronic migraine affects the functionality of patients severely. In a community-based study conducted by Bigal et al. (6), more than $50 \%$ of chronic migraine patients were unable to do houseworks for at least 5 days per month, while in patients with episodic migraines, this rate was found to be $24.3 \%$. 36.9\% of chronic migraine patients and $9.5 \%$ of episodic migraine patients reported insufficient participation in family activities. The average number of head pain days per month of the patients evaluated in our study was $23.9 \pm 5.7$ days/month and $4.2 \%$ of the patients had been admitted to the emergency department for headache at least 1 time in the last 1 year. Almost half of the patients have a history of applying to the emergency department and the average number of monthly painful days indicate serious disability.

In the study conducted by Bigal et al. (6), 20.2\% of patients with chronic migraine were previously diagnosed with chronic migraine and $13.9 \%$ were diagnosed with drug overuse headache or rebound headache. $65.5 \%$ of the patients evaluated in our study had previously been diagnosed with migraines and there were no patients diagnosed with chronic migraines in our group. These rates indicate the problem with diagnosis of chronic migraine.

Although migraine has been associated with many psychiatric disorders, few studies have proven the effect of psychiatric comorbidity on migraine progression. In the study by Mathew et al. (23), patients were evaluated with the Minnesota Multiphasic Personality Inventory. Episodic migraines were accompanied by $12.2 \%$ chronic daily headaches and $70.5 \%$ personality disorder. The most common psychiatric disorders associated with chronic daily headaches were hysteria, hypochondriasis and depression. When patients were evaluated with depression scale, depression was found to be $5 \%$ in episodic migraine and $46 \%$ in chronic headache. The presence of stress detected by behavioral therapists or psychiatrists was $30.4 \%$ in episodic migraine and $67.2 \%$ in chronic daily headaches. This study shows that psychiatric comorbidity has an effect on the progression of headache. Our study showed the presence of depression with a $62.6 \%$ depression scale in line with the literature. $8.6 \%$ of the patients had a history of psychiatric illness (depression, anxiety, panic attacks) before, although this rate is significantly lower than the ratio in the literature, this decrease can be explained by the lack of application of the patients to the psychiatrist, as $91.4 \%$ of the patients reported the presence of stress at the time of application that would affect their daily life activities.

In a community-based study by Le et al. (24) on 46418 people, 20-30\% comorbid disease was observed in migraine patients. Accompanying comorbid diseases include cardiovascular diseases, stroke, musculoskeletal diseases, low back pain, neck pain, history of head and neck trauma, thyroid gland disease, meniere, allergic disease, asthma, epilepsy, kidney stone, osteoarthritis, scoliosis, osteoporosis, fibromyalgia, autoimmune diseases, systemic lupus erythematosus, psoriasis, rheumatoid arthritis and collagen tissue diseases were found in higher rates in patients with migraine than in patients without migraine. There was no significant difference in the incidence of diabetes mellitus, coronary artery disease, juvenile arthritis and ankylosing spodilitis among the group with and without migraine. In our study, $56.1 \%$ of patients had at least 1 comorbid disease. The presence of frequent comorbid disease is important for chronic headache and treatment choice. Accompanying comorbid diseases are summarized in Table 2 according to their incidence.

According to the study conducted by Robbins Headache Clinic on 494 migraine diagnosed patients, migraine was predisposed with $62 \%$ stress, $43 \%$ weather changes, $40 \%$ meal skipping, 38\% 
sunlight, 31\% insomnia, 30\% dietary factors, 29\% perfume, $26 \%$ smoking, 24\% stress, 24\% sleep, $15 \%$ exercise and 5\% sexual activity (25). In this study, the factors that predispose the headaches of the patients were summarized in Table 4 in order of frequency. The patients evaluated in our study had many predisposing factors in line with the literature, with the exception being predisposition with exercise and sexual activity, which was detected at low rates.

Unnecessary excessive analgesic use has been cited as a poor prognostic factor for migraines. In many community-based studies, it was observed that migraine was the most risky group of patients in terms of drug overuse headache development. In the American Migraine Prevalence and Prevention study, the increased risk of developing chronic migraine was 2 times higher in migraine patients using barbiturates and opiates. In the study, it was found that triptans did not increase risk in terms of episodic migraine transformation, but initially facilitated transformation in headache high frequency migraines, and there was no risk in NSAIDs (26). In the study by Mathew et al. (23); caffeine combined analgesic, narcotic analgesic and ergotamine use was $6 \%$ in the group with episodic headache and $52.4 \%$ in the group with chronic headache. In the Norwegian Head-HUNT study, 39.3\% of patients who used analgesic almost daily developed drug overuse headaches (27). Bigal et al. (6) in a study conducted by, $24.8 \%$ of patients with episodic migraines and $31.6 \%$ of patients with chronic migraines were using migraine-specific analgesics for attack treatment. The use of one or more non-prescription analgesics was observed in $82.6 \%$ of chronic migraine patients and the average monthly analgesic use was determined to be 15.9 days/month. In our study, the use of analgesics in $71.2 \%$ of patients meeting the analgesic overuse criteria was determined. It was found that $41.7 \%$ of the patients used NSAIDs, $10.8 \%$ of ergotamine, $1.4 \%$ of triptan and $17.3 \%$ of these drugs in different combinations. In accordance with the literature, excessive use of analgesics accompanies chronic migraine at a high rate and most of the analgesics are used without a prescription. This is an indication that high levels of inappropriate analgesic use in

\begin{tabular}{|l|l|l|l|}
\hline \multicolumn{4}{|l|}{ Table 4. Predisposing factors and their incidence } \\
\hline Predisposing factor & Frequency & $\begin{array}{l}\text { Predisposing } \\
\text { factor }\end{array}$ & Frequency \\
\hline Environmental factors & $\% 91.4$ & $\begin{array}{l}\text { Hormonal } \\
\text { change }\end{array}$ & $\% 57.6$ \\
\hline Stress & $\% 86.3$ & Dietary factors & $\% 33.1$ \\
\hline Sleep disorder & $\% 86.3$ & $\begin{array}{l}\text { Physical } \\
\text { activity }\end{array}$ & $\% 0.7$ \\
\hline Skipping meals & $\% 85.6$ & Sexual activity & $\% 0$ \\
\hline
\end{tabular}

chronic migraine patients are one of the common problems. In a study conducted by Bigal et al. (6), $40 \%$ of patients with chronic migraines received prophylactic treatment and the proportion of patients receiving regular treatment was 33.3\%. In line with the literature, $46 \%$ of the patients evaluated in our study did not receive prophylactic treatment, 36\% did not respond to the prophylactic treatment they had previously received, and $18 \%$ reported that they had partially benefited from the prophylactic treatment they had previously received. Prophylactic treatment was initiated for all patients, 120 patients were evaluated and $72.5 \%$ of patients received positive response after one year of follow-up. Information on prophylactic treatment is summarized in Table 3. In classic agents used in migraine prophylaxis, treatment response was similar to that of episodic migraine. There was a significant decrease in headache frequency and severity in 51.9\% of patients after dry needle treatment and $70.1 \%$ of patients after botulinum toxin a treatment.

\section{CONCLUSION}

Chronic migraine seriously worsens the quality of life of the patients due to its high number of painful days and its severity. Chronic migraine is often accompanied by overuse of analgesics, but it is difficult to treat and follow-up in neurology practice and a high rate of recurrence develops after treatment. In our study, female gender, low socioeconomic level, low educational level, analgesic overuse, obesity and associated psychiatric illness were found as risk factors for chronic headache. Risk factors are determinant in terms of the method to be used in treatment and in terms of prognosis. In chronic migraine, similar treatment response was received with agents used in episodic migraine prophylaxis. In treatment of resistant cases, approximately half of the patients were treated with dry needle in addition to prophylactic treatment, and up to $70 \%$ responded with Botulinum toxin $\mathrm{A}$ administration. This suggests that dry needle administration and Botulinum toxin A treatment, especially in treatment resistant cases, lead to a significant increase in clinical response and may be used as the main treatment option in resistant cases. As a result of rapid detoxification and transition therapy in patients with analgesic overuse, similar treatment responses were received in the group of patients without analgesic overuse. We attribute the significant increase in patients' response rates to treatment after application to increasing patient compliance by regular follow-up, use of appropriate treatment time at appropriate doses and time. 


\section{Ethics}

Ethics Committee Approval: Ethics Committee of University of Health Sciences Turkey Okmeydanı Education and Research Hospital dated 14.04.2015 and numbered 303.

Informed Consent: Our study was conducted retrospectively and patient consent was not obtained.

Peer-review: Externally peer-reviewed.

\section{Authorship Contributions}

Analysis or Interpretation: B.K., O.A., S.Ü., Literature Search: B.K., O.A., S.Ü., Writing: B.K., O.A., S.Ü.

Conflict of Interest: No conflict of interest was declared by the authors.

Financial Disclosure: The authors declared that this study received no financial support.

\section{REFERENCES}

1. Headache Classification Committee of the International Headache Society (IHS). The International Classification of Headache Disorders, 3rd edition (beta version). Cephalalgia 2013;33:629.

2. Stewart WF, Shechter A, Rasmussen BK. Migraine prevalance. A review of population-based studies. Neurology 1994;44:17-23.

3. Lipton RB, Bigal ME, Diamond M, Freitag F, Reed ML, Stewart WF, et al. Migraine prevalence, disease burden, and the need for preventive therapy. Neurology 2007;68:343-9.

4. Bigal ME, Serrano D, Buse D, Scher A, Stewart WF, Lipton RB. Acute migraine medications and evolution from episodic to chronic migraine: a longitudinal population-based study. Headache 2008;48:1157-68.

5. Natoli JL, Manack A, Dean B, Butler Q, Turkel CC, Stovner L, et al. Global prevalance of chronic migraine: a systematic review. Cephalalgia 2010;30:599.

6. Bigal ME, Serrano D, Reed M, Lipton RB. Chronic migraine in the population: burden, diagnosis, and satisfaction with treatment. Neurology 2008;71:559-66.

7. Guitera V, Muñoz P, Castillo J, Pascual J. Quality of life in chronic daily headache: a study in a general population. Neurology 2002;58:1062-5.

8. Katsarava Z, Muessig M, Dzagnidze A, Fritsche G, Diener HC, Limmroth V. Medication overuse headache: rates and predictors for relapse in a 4-year prospective study. Cephalalgia 2005;25:12-5.

9. Schwedt TJ. Chronic migraine. BMJ 2014;348:1416.

10. Schwedt TJ, Dodick DW. Advanced neuroimaging of migraine. Lancet Neurol 2009;8:560-8.
11. Aurora SK. Is chronic migraine one end of a spectrum of migraine or a separate entity?. Cephalalgia 2009;29:597-605.

12. Scher Al, Stewart WF, Ricci JA, Lipton RB. Factors associated with the onset and remission of chronic daily headache in a population-based study. Pain 2003;106:81-9.

13. Scher Al, Midgette LA, Lipton RB. Risk factors for headache chronification. Headache 2008;48:16-25.

14. Bigal M. Migraine chronification--concept and risk factors. Discov Med 2009;8:145-50

15. Castillo J, Muñoz P, Guitera V, Pascual J. Kaplan Award 1998 Epidemiology of chronic daily headache in the general population. Headache 1999;39:190-6.

16. Lu SR, Fuh JL, Chen WT, Juang KD, Wang SJ. Chronic daily headache in Taipei, Taiwan: prevalence, follow-up and outcome predictors. Cephalalgia 2001;21:980-6

17. Scher Al, Stewart WF, Liberman J, Lipton RB. Prevalence of frequent headache in a population sample. Headache 1998;38:497-506.

18. Hagen K, Vatten L, Zwart JA, Krokstad S, Bovim G. Low socioeconomic status is associated with increased risk of frequent headache: a prospective study of 22718 adults in Norway. Cephalalgia 2002;22:672-

19. Peterlin BL, Rosso AL, Williams MA, Rosenberg JR, Haythornthwaite JA, Merikangas KR, et al. Episodic migraine and obesity and the influence of age, race and sex. Neurology 2013; 81:1314-21.

20. Vo M, Ainalem A, Qiu C, Peterlin BL, Aurora SK, Williams MA. Body mass index and adult weight gain among reproductive age women with migraine. Headache 2011;51:559-69.

21. Bigal ME, Lipton RB. Obesity is a risk factor for transformed migraine but not chronic tension type headache. Neurology 2006;67:252-7.

22. Tietjen GE, Peterlin BL, Brandes JL, Hafeez F, Hutchinson S, Martin VT, et al. Depression and anxiety: effect on the migraine obesity relationship. Headache 2007;47:866-75.

23. Mathew NT, Stubits E, Nigam MP. Transformation of episodic migraine into daily headache: analysis of factors. Headache 1982;22:66-8.

24. Le H, Tfelt-Hansen P, Russell MB, Skytthe A, Kyvik KO, Olesen J. Comorbidity of migraine with somatic disease in a large population-based study. Cephalalgia 2011;31:43-64.

25. Robbins L. Precipitating factors in migraine: a retrospective review of 494 patients. Headache 1994;34:214-6.

26. Diamond S, Bigal ME, Silberstein S, Loder E, Reed M, Lipton RB. Patterns of diagnosis and acute and preventive treatment for migraine in the United States: results from the American Migraine Prevalence and Prevention study [published correction appears in Headache. 2007 Oct;47:1365]. Headache 2007;47:355-63.

27. Zwart JA, Dyb G, Hagen K, Svebak S, Stovner LJ, Holmen J. Analgesic overuse among subjects with headache, neck, and low-back pain. Neurology 2004;62:1540-4. 